\title{
Effects of Two $\alpha$-Glucosidase Inhibitors, Acarbose, and BAY-m-1099, on Intestinal Microflora, Cecal Properties, Body-Weight Gains, Serum Cholesterol and Serum Lipids of Rats
}

\author{
Yoshimi Benno, ${ }^{1}$ Kimiko Endo, ${ }^{1}$ Nobue Shiragami ${ }^{1}$ \\ and Tomotari MitsuokA ${ }^{1,2}$ \\ ${ }^{1}$ The Institute of Physical and Chemical Research, Wako, Saitama, 351-01, \\ ${ }^{2}$ Department of Biomedical Science, Faculty of Agriculture, The University of Tokyo, \\ Bunkyo-ku, Tokyo, 113
}

(Received for publication, July 27, 1988)

\begin{abstract}
Effects of two $\alpha$-glucosidase inhibitors, Acarbose and BAY-m-1099, on the intestinal microflora, cecal weight, cecal ammonia concentration, cecal $\mathrm{pH}$, body-weight gains, and levels of serum cholesterol and lipids of Fischer male rats were determined. The cecal microflora of rats fed Acarbose-supplemented diets showed a reduction of Enterobacteriaceae and increases of lecithinase-negative Clostridium spp. and Peptostreptococcus spp. by feeding day (FD) 14. By FD 28, the numbers of Bifidobacterium spp. in the Acarbose groups were higher than those in the control and BAY-m-1099 groups. Increasing numbers of Staphylococcus spp. were observed in the cecum of rats fed Acarbose (the volume of intake, $40 \mathrm{mg} / 100 \mathrm{~g}$ of feed)- and BAY-m1099-supplemented diets. The weight of cecum in the Acarbose groups was significantly increased when compared with those in the other groups. The ammonia concentration and $\mathrm{pH}$ values in the cecum of rats fed Acarbose diets were significantly lower than those in the other groups. The body-weight gains in the Acarbose groups by FD 28 were significantly lower than those in the BAY-m-1099 and control groups. Significantly decreased levels of total cholesterol, LDL cholesterol, triglycerides, and phospholipids were observed in the Acarbose groups by FD 28. These findings indicated that inhibition of sugar absorption in the small intestine of rats influenced the composition of intestinal microflora, body-weight gains, differences in cecal weight, cecal $\mathrm{pH}$, and cecal ammonia concetration, bodyweight gains, and the levels of serum cholesterol and lipids.
\end{abstract}

Key words : $\alpha$-glucosidase inhibitor; intestinal microflora; cecal ammonia; serum cholesterol and lipids 
Pseudo-oligosaccharide (Acarbose, BAY-g-5421) and deoxynojirimycin derivative (BAY-m-1099) suppress the digestion of carbohydrates in parts of the small intestine by inhibiting $\alpha$-glucosidase activity $(11,12)$. Such reductions of carbohydrate absorption, i.e. sucrose, maltose, and isomaltose, from the small intestine may cause an increased concentration of carbohydrates and change the composition of the microflora in the large intestine. Acarbose (BAY-g-5421) inhibited carbohydrate absorption in all parts of the small intestine, while BAY-m-1099 did not inhibit it in the ileum. Excretion of carbohydrates in the feces was increased by Acarbose, but not changed by BAY-m-1099. It is of interest to determine what effects, if any, these two $\alpha$-glucosidase inhibitors exert on the intestinal microflora.

The present study was undertaken to define the different effects of Acarbose and BAY-m-1099 on the intestinal microflora, cecal weights, cecal water contents, cecal $\mathrm{pH}$, cecal ammonia concentration, body-weight gains, serum cholesterol and serum lipids of rats.

\section{MATERIALS AND METHODS}

Animals. Eighty Fischer male rats (Charles River Japan Inc.) with an average initial weight of $188 \mathrm{~g}$ were used. The rats were kept in $34 \times 45 \times 19 \mathrm{~cm}$ cages with five rats per cage. All animals were fed ad libitum a standard diet (MF, Oriental Yeast Co., Ltd., Japan) and water for 1 week before the experiment. The composition of MF consists of crude protein, $24 \%$; fat, $5.1 \%$; ash, $6.2 \%$; crude fiber, $3.2 \%$; soluble non-protein compounds, $54.5 \%$; vitamins and others, $0.16 \%$; and water, $7.0 \%$. The rats were then assigned to four groups: group $\mathrm{A}$ fed $\mathrm{MF}$ diet alone as a control group; group B fed MF with Acarbose $(20 \mathrm{mg} / 100 \mathrm{~g}$ of MF); group C fed MF with Acarbose (40 mg/100 g); and group D fed MF with BAY-m1099 (40 mg/100 g). Acarbose and BAY-m-1099 were supplied by Bayer Yakuhin, Ltd., Osaka. Each group consisted of 20 rats. All experimental diets were given to each rat for 28 days. The animal room was kept at $24 \pm 1^{\circ} \mathrm{C}$; relative humidity was $55 \pm 5 \%$; light and dark periods were at 12 -hr intervals. Cages and bedding were changed once a week. Body-weight gains were computed on a weekly basis.

Sampling procedures. After feeding days (FD) 14 and 28, the microflora of the small intestine (mainly the upper part of the ileum) and cecum of rats in each group was examined. Fresh samples of the small intestine and cecum were collected from 10 rats on FD 14 and 28, respectively.

Bacterial analysis. The method for bacterial analysis of the intestinal microflora in this study was essentially the same as that of Mitsuoka et al $(6,7)$. After thorough mixing, a series of 10 -fold dilutions $\left(10^{-1}\right.$ to $\left.10^{-7}\right)$ was made in anaerobic diluents $(6)$. From appropriate dilutions, $0.05 \mathrm{ml}$ aliquots were spread onto four non-selective agar plates [modified medium 10 (M10) with $10 \%$ cecal contents of rats for fastidious anaerobes (6); modified Eggerth-Gagnon (EG) agar for anaerobes (7); glucose blood liver (BL) agar for anaerobes (7); trypticase soy blood (TS) agar (BBL Microbiology System, Cockeysville, Md) for aerobes (7)] as well as eleven selective agar plates [bifidobacteria-selective (BS) agar for Bifidobacterium spp. (7); eubacteria- 
selective (ES) agar for Eubacterium spp. (6); neomycin-brilliant green-taurocholateblood (NBGT) agar Bacteroides spp. (7); neomycin-Nagler (NN) agar for lecithinasepositive Clostridium spp. (7); modified veillonella-selective (VS) agar for Veillonella spp. and Megasphaera sp. (7); modified lactobacilli-selective (LBS) agar for Lactobacillus spp. (7); triphenyltetrazolium chloride-acridine orange-thallous sulfate aesculin crystal violet (TATAC) agar for Enterococcus spp. and Streptococcus spp. (7); phenylethylalcohol egg yolk suspension (PEES) agar for Staphylococcus spp. (7); potato dextrose (PD) agar (Difco, Detroit) for yeasts and molds (7); deoxycholate hydrogen sulfide lactose (DHL) agar (Eiken, Tokyo) for Enterobacteriaceae (7); nalixidic acid cetrimide (NAC) agar (Eiken) for Pseudomonas aeruginosa]. Modified medium 10 was used in the "Plate-in-bottle" method $(8)$ at $37^{\circ} \mathrm{C}$ for 5 days. Eight agar plates of EG, BL, BS, NBGT, ES, NN, VS, and LBS were incubated at $37^{\circ} \mathrm{C}$ for 3 days in an anaerobic steel-wool jar (6) filled with an atmosphere of oxygen-free $\mathrm{CO}_{2}$. Three agar plates of TATAC, PEES, and NAC were incubated aerobically at $37^{\circ} \mathrm{C}$ for $48 \mathrm{hr}$; TS and DHL agar plates were incubated at $37^{\circ} \mathrm{C}$ for $24 \mathrm{hr}$.

After incubation, each plate was examined for bacterial colonies. The identification of 16 bacterial groups, yeasts, and molds was performed with colonial and cellular morphologies, gram-reaction, spore formation, and aerobic growth. For the bacterial species identified, the bacterial count per gram of wet intestinal contents was calculated and converted into a logarithmic equivalent. The total viable count was calculated from the sum of the counts of each bacterial species.

Cecal weight and water content. After each animal on FD14 and FD28 was killed, the cecum was removed and weighed. The cecal water content was calculated as the wet weight minus the dry weight of contents. The drying of cecal contents was performed by the vacuum drying oven (VO-3-5, Shimizu Rikagaku Co., Ltd., 'Tokyo).

Cecal pH. The cecal $\mathrm{pH}$ was measured in situ as the cecum was dissected, using a combination electrode (Horiba Co., Ltd., Tokyo).

Ammonia concentration in cecum. The ammonia concentration in the cecum of rats was determined by use of an ammonia test kit (Wako Pure Chemical Industries, Ltd., Osaka).

Serum cholesterol and lipids. Serum cholesterol (1) and triglycerides (5) were measured by enzyme techniques. HDL and LDL cholesterol were measured by use of the precipitation method with heparin, $\mathrm{Ca}^{2+}$, and an anion-exchange resin (9, 10). Phospholipids were determined by a combined enzymatic method using phospholipase $\mathrm{D}$, choline oxidase and peroxidase (15).

\section{RESULTS}

Effects of Acarbose and BAY-m-1099 on the Small Intestine and Cecal Microflora of Rats

The compositions of the small intestinal and cecal microflora of 10 rats in four groups by FD 14 are shown in Table 1. In the small intestine, a significant reduction of Enterobacteriaceae $(P<0.05)$ was found in the Acarbose $40 \mathrm{mg}$ group compared with the control group. The cecal microflora of rats fed Acarbose (20 mg and 
Table 1. Effects of Acarbose and BAY-m-1099

\begin{tabular}{|c|c|c|c|c|}
\hline \multirow{2}{*}{ Intestinal flora } & \multicolumn{2}{|c|}{ Group A } & \multicolumn{2}{|c|}{ Group B } \\
\hline & Small intestine & Cecum & Small intestine & Cecum \\
\hline Total bacteria ${ }^{a}$ & $8.8 \pm 0.3^{b}(100)^{c}$ & $10.2 \pm 0.1(100)$ & $8.9 \pm 0.2 \quad(100)$ & $10.0 \pm 0.3(100)$ \\
\hline \multicolumn{5}{|l|}{ Anaerobic bacteria } \\
\hline Bacteroidaceae & $(10)$ & $10.0 \pm 0.3(100)$ & $4.3 \pm 1.8^{d}(70)$ & $9.8 \pm 0.5 \quad(100)$ \\
\hline Eubacterium spp. & $(0)$ & $7.3 \pm 1.6(40)$ & $(0)$ & $8.3 \pm 0.5 \quad(80)$ \\
\hline Peptostreptococcus spp. & $0)$ & $8.1 \pm 0.6(80)$ & $(10)$ & $9.1 \pm 0.7$ \\
\hline Bifidobacterium spp. & $(0)$ & $6.8 \pm 2.9(30)$ & $(0)$ & $(0)$ \\
\hline $\begin{array}{l}\text { Lecithinase-positive } \\
\text { Clostridium spp. }\end{array}$ & $2.4 \pm 0.1 \quad(20)$ & $2.7 \pm 0.4(60)$ & $(10)$ & $2.9 \pm 0.7$ \\
\hline $\begin{array}{l}\text { Lecithinase- } \\
\text { Clostridium }\end{array}$ & $3.2 \pm 0.6 \quad(80)$ & $6.3 \pm 1.8(100)$ & $2.9 \pm 0.7 \quad(40)$ & $7.2 \pm 1.5$ \\
\hline Curved rods & $(0)$ & $9.3 \pm 0.5(60)$ & $0)$ & $(0)$ \\
\hline Spiral-shaped rods & $0)$ & $9.3 \quad(10)$ & $0)$ & \\
\hline Fusiform bacteria & $0)$ & $9.0 \pm 0.5(100)$ & $0)$ & $9.0 \pm 0.6(100)$ \\
\hline \multicolumn{5}{|l|}{ Aerobic bacteria } \\
\hline Lactobacillus spp. & $8.8 \pm 0.3 \quad(100)$ & $9.0 \pm 0.2(100)$ & $8.9 \pm 0.2 \quad(100)$ & $9.2 \pm 0.3 \quad(100)$ \\
\hline Enterobacteriaceae & $4.7 \pm 1.0(100)$ & $5.7 \pm 0.5(100)$ & $4.3 \pm 1.0 \quad(100)$ & $5.1 \pm 0.5^{e}(100)$ \\
\hline Streptococcus spp. & $4.4 \pm 0.9 \quad(100)$ & $5.0 \pm 0.4(100)$ & $4.0 \pm 0.3$ & $5.3 \pm 0.9 \quad(100)$ \\
\hline Staphylococcus spp. & $6.0 \pm 0.7$ & $6.2 \pm 0.8(100)$ & $5.5 \pm 0.6 \quad(100)$ & $5.9 \pm 0.7$ \\
\hline Corynebacterium spp. & $4.9 \quad(10)$ & $6.6 \quad(10)$ & $6.3 \quad(10)$ & $(10)$ \\
\hline Yeasts & $(0)$ & $(0)$ & $(10)$ & $(0)$ \\
\hline Molds & $2.3 \pm 0.3 \quad(30)$ & $2.5 \pm 0.2(30)$ & $2.4 \pm 0.1 \quad(20)$ & $2.6 \pm 0.5(40)$ \\
\hline
\end{tabular}

$a:$ No veillonella and megasphaera were detected.

${ }^{b}$ : Mean of $\log _{10}$ no. of bacteria/g of intestinal content \pm SD.

$c$ : Frequencies of occurrence ( $\%)$.

a : Statistically significant at the $P<0.05$ level when compared with the bacterial number in

$e$ : Statistically significant at the $P<0.05$ level when compared with the bacterial number in

$f$ : Statistically significant at the $P<0.001$ level when compared with the bacterial number in

${ }^{\circ}$ : Statistically significant at the $P<0.001$ level when compared with the bacterial number in

$40 \mathrm{mg}$ per $100 \mathrm{~g}$ of feed, respectively)-supplemented diets showed a significant reduction of Enterobacteriaceae $(P<0.05)$ and an increase of lecithinase negative $($ Clostridium spp. and Peptostreptococcus spp.

Decreased numbers of Lactobacillus spp. $(P<0.05)$ and increased numbers of Staphylococcus spp. $(P<0.001)$ were found in the small intestine of rats fed BAY-m1099-supplemented diets. Significantly increased numbers of Staphylococcus spp. $(P<0.001)$ were found in the cecum of the BAY-m-1099 group compared with that of controls. Additionally, the intake of BAY-m-1099 also induced the significantly increased numbers of fusiform bacteria $(P<0.05)$ and Staphylococcus spp. $(P<0.001)$ compared with that of Acarbose.

The small intestinal and cecal microflora of 10 rats in each group by FD 28 is listed in Table 2. Significant reductions of Enterobacteriaceae $(P<0.05)$ in the small intestine of all animals fed Acarbose- and BAY-m-1099-supplemented diets were found in the present study. The numbers of Staphylococcus spp. from the cecum of animals in the Acarbose-40 mg and BAY-m-1099 groups were significantly higher than those in the controls. The counts of Bifidobacterium spp., Peptostreptococcus spp., 
on the intestinal flora of rats after two weeks

\begin{tabular}{|c|c|c|c|c|c|}
\hline \multicolumn{4}{|c|}{ Group C } & \multicolumn{2}{|c|}{ Group D } \\
\hline \multicolumn{2}{|c|}{ Small intestine } & \multicolumn{2}{|c|}{ Cecum } & Small intestine & Cecum \\
\hline $8.6 \pm 0.3$ & $(100)$ & $10.3 \pm 0.3$ & $(100)$ & $8.4 \pm 0.4 \quad(100)$ & $10.2 \pm 0.1 \quad(100)$ \\
\hline \multirow[t]{2}{*}{$4.4 \pm 1.7$} & $(60)$ & $9.7 \pm 0.4$ & $(100)$ & $2.9 \pm 1.0 \quad(40)$ & $9.9 \pm 0.3 \quad(100)$ \\
\hline & $(0)$ & $8.7 \pm 0.5$ & ( 50$)$ & $(0)$ & $6.4 \pm 2.5(60)$ \\
\hline \multirow[t]{3}{*}{7.0} & $(10)$ & $9.5 \pm 0.6$ & $(100)$ & $(0)$ & $8.8 \pm 0.8(80)$ \\
\hline & $(0)$ & & $(0)$ & $(0)$ & $5.9 \pm 4.4 \quad(20)$ \\
\hline & $(0)$ & & $(0)$ & $3.2 \pm 1.6 \quad(20)$ & $2.8 \pm 0.3(80)$ \\
\hline \multirow[t]{4}{*}{3.4} & $(70)$ & $7.2 \pm 0.8$ & $(100)$ & $2.6 \pm 0.5(50)$ & $6.6 \pm 1.4 \quad(100)$ \\
\hline & $(0)$ & $9.3 \pm 0.8$ & $(100)$ & $(0)$ & $9.6 \pm 0.4 \quad(70)$ \\
\hline & $(0)$ & & $(0)$ & $0)$ & $(0)$ \\
\hline & $(0)$ & $8.9 \pm 0.5$ & $(100)$ & $(0)$ & $9.4 \pm 0.5 \quad(100)$ \\
\hline $8.6 \pm 0.3$ & $(100)$ & $9.1 \pm 0.3$ & $(100)$ & $8.4 \pm 0.3^{d}(100)$ & $9.0 \pm 0.2 \quad(100)$ \\
\hline $3.7 \pm 0.9^{d}$ & $(100)$ & $4.8 \pm 1.0^{e}$ & $(100)$ & $4.1 \pm 0.5$ & $5.2 \pm 0.7 \quad(100)$ \\
\hline $3.9 \pm 0.5$ & $(100)$ & $5.8 \pm 0.7$ & $(100)$ & $4.1 \pm 0.3 \quad(100)$ & $4.8 \pm 0.6 \quad(100)$ \\
\hline \multirow[t]{3}{*}{$5.9 \pm 0.8$} & $(100)$ & $5.9 \pm 0.9$ & $(100)$ & $7.3 \pm 0.3^{f}(100)$ & $7.8 \pm 0.6^{g}(100)$ \\
\hline & $(0)$ & & $(0)$ & $6.5 \pm 0.1 \quad(20)$ & $6.8 \pm 0.6 \quad(20)$ \\
\hline & $(0)$ & 2.2 & $(10)$ & $(0)$ & $2.9 \pm 1.3(50)$ \\
\hline 2.5 & $(10)$ & $2.3 \pm 0.4$ & $(30)$ & $(10)$ & $2.4 \quad(10)$ \\
\hline
\end{tabular}

the small intestine of group A.

the cecum of group A.

the small intestine of group A.

the cecum of group A.

and lecithinase-negative Clostridium spp. in the cecum of rats in both Acarbose groups were higher than those in control and BAY-m-1099 groups.

The numbers of fusiform bacteria and Enterobacteriaceae from the cucum of rats fed BAY-m-1099-supplemented diets were significantly higher than those given the Acarbose diet, whereas the frequency of occurrence of Bifidobacterium spp. in the rats fed BAY-m-1099-supplemented diets was lower than that in the animals fed Acarbose.

Effects of Acarbose and BAY-m-1099 on the Body Weight of Rats

The gains of body weight during feeding of Acarbose and BAY-m-1099 were computed on a weekly basis as given in Fig. 1. After FD 14, no significant bodyweight gains were found in each group. By FD 28, body weights of rats given food containing $20 \mathrm{mg}$ and $40 \mathrm{mg}$ of Acarbose were significantly lower than those of rats in the BAY-m-1099 and control groups. 
Table 2. Effects of Acarbose and BAY-m-1099

\begin{tabular}{|c|c|c|c|c|}
\hline \multirow{2}{*}{ Intestinal flora } & \multicolumn{2}{|c|}{ Group A } & \multicolumn{2}{|c|}{ Group B } \\
\hline & Small intestine & Cecum & Small intestine & Cecum \\
\hline Total bacteria $^{a}$ & $8.6 \pm 0.5^{b}(100)^{c}$ & $10.5 \pm 0.2(100)$ & $8.6 \pm 0.4 \quad(100)$ & $\overline{10.4} \pm 0 . \overline{(100)}$ \\
\hline \multicolumn{5}{|l|}{ Anaerobic bacteria } \\
\hline Bacteroidaceae & $4.1 \pm 2.1 \quad(80)$ & $10.1 \pm 0.4(100)$ & $3.9 \pm 1.0 \quad(40)$ & $10.2 \pm 0.3(100)$ \\
\hline Eubacterium spp. & $(0)$ & $7.8 \pm 2.6(40)$ & $-\quad(0)$ & $8.3 \pm 1.1(70)$ \\
\hline Peptostreptococcus spp. & $7.7 \pm 0.8 \quad(30)$ & $9.0 \pm 0.7(100)$ & $0)$ & $9.6 \pm 0.5(100)$ \\
\hline Bifidobacterium spp. & $2.3 \quad(10)$ & $2.7 \pm 0.2(30)$ & $0)$ & $9.1 \pm 0.9(30)$ \\
\hline $\begin{array}{l}\text { Lecithinase-positive } \\
\text { Clostridium spp. }\end{array}$ & $(0)$ & $2.8 \pm 0.4(40)$ & $(0)$ & $4.1 \pm 0.7(30)$ \\
\hline $\begin{array}{l}\text { Lecithinase-negative } \\
\text { Clostridium spp. }\end{array}$ & $3.6 \pm 0.9(40)$ & $5.9 \pm 1.2(90)$ & $3.2 \pm 1.5 \quad(80)$ & $7.8 \pm 1.2(100)$ \\
\hline Curved rods & $(10)$ & $9.7 \pm 0.2(90)$ & & $9.0 \pm 0.7(50)$ \\
\hline Spiral-shaped rods & $(0)$ & $(0)$ & $0)$ & $8.8 \quad(10)$ \\
\hline Fusiform bacteria & $(0)$ & $9.7 \pm 0.4(100)$ & $0)$ & $9.4 \pm 0.5(60)$ \\
\hline \multicolumn{5}{|l|}{ Aerobic bacteria } \\
\hline Lactobacillus spp. & $8.6 \pm 0.4 \quad(100)$ & $9.3 \pm 0.5(100)$ & $8.6 \pm 0.4 \quad(100)$ & $9.2 \pm 0.6(100)$ \\
\hline Enterobacteriaceae & $4.7 \pm 0.8 \quad(100)$ & $5.2 \pm 0.5(100)$ & $4.0 \pm 0.5^{d}(100)$ & $5.0 \pm 0.5(100)$ \\
\hline Streptococcus spp. & $5.4 \pm 0.9(100)$ & $5.3 \pm 0.6(100)$ & $4.2 \pm 0.6 \quad(100)$ & $4.4 \pm 0.9(100)$ \\
\hline Staphylococcus spp. & $5.5 \pm 0.7 \quad(100)$ & $5.0 \pm 1.6(100)$ & $5.9 \pm 1.0 \quad(100)$ & $5.9 \pm 0.5(100)$ \\
\hline Corynebacterium spp. & $-(0)$ & $(0)$ & $2.3 \quad(10)$ & $5.4 \pm 1.1(20)$ \\
\hline Yeasts & $(0)$ & $(0)$ & $(0)$ & $2.5 \pm 0.7(40)$ \\
\hline Molds & $2.1 \quad(10)$ & $2.4 \pm 0.1(70)$ & $2.1 \pm 0.1 \quad(20)$ & $2.2 \pm 0.2(30)$ \\
\hline $\begin{array}{l}a: \text { No veillonella } \\
b: \text { Mean of } \log _{10} \\
c: \text { Frequencies o } \\
{ }^{a}: \text { Statistically } \\
e: \text { Statistically si }\end{array}$ & $\begin{array}{l}\text { nd megasphaera } \\
\text { cof bacteria/g of } \\
\text { ccurrence }(\%) \text {. } \\
\text { ificant at the } P< \\
\text { ificant at the } P<\end{array}$ & $\begin{array}{l}\text { re detected. } \\
\text { testinal content }\end{array}$ & SD. & \\
\hline
\end{tabular}

\section{Effects of Acarbose and BAY-m-1099 on Cecal Contents, Cecal pH, and Ammonia Concentration in the Cecum}

Effects of Acarbose and BAY-m-1099 on the cecal weight, cecal pH, cecal water content, and ammonia concentration in the cecum are shown in Table 3. The weight of the cecum in the Acarbose groups was significantly increased when compared with those in the BAY-m-1099 and control groups. The heaviest cecum was found in the Acarbose $40 \mathrm{mg}$ group. The water content in the cecum of rats in the Acarbose- $40 \mathrm{mg}$ group for four weeks was higher than those of other groups. The cecal $\mathrm{pH}$ in the Acarbose- $40 \mathrm{mg}$ group was significantly lower than those in the BAY-m-1099 and control groups. The ammonia concentrations in the cecum of rats in the Acarbose groups by FD14 and FD28 were significantly lower than those in the BAY-m-1099 and control groups.

\section{Effects of Acarbose and BAY-m-1099 on Serum Cholesterol and Serum Lipids of Rats}

Effects of Acarbose and BAY-m-1099 on the levels of serum cholesterol and lipids of the rats are indicated in Table 4. The levels of total cholesterol, LDL cholesterol, and phospholipids of rats fed Acarbose- $40 \mathrm{mg}$-supplemented diet were significantly 
on the intestinal flora of rats after four weeks

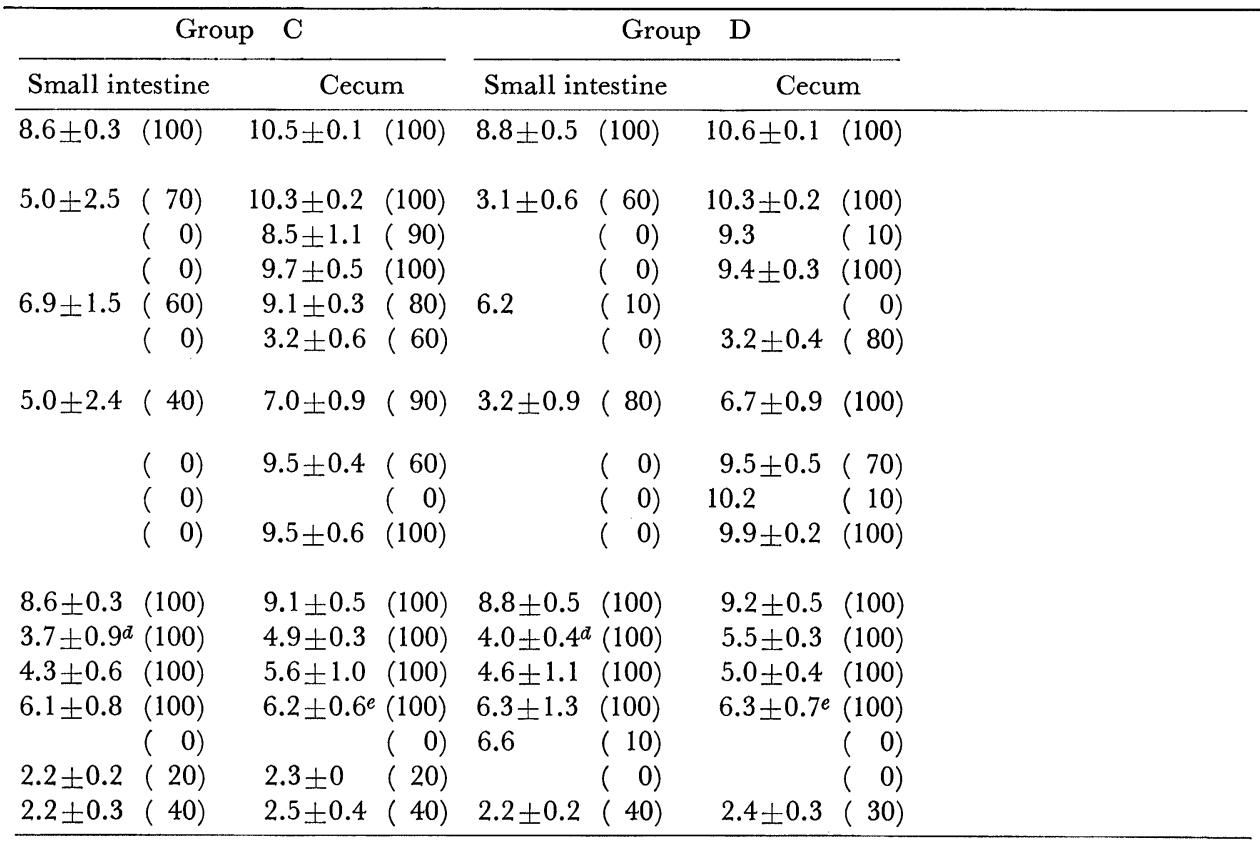

the small intestine of group A.

the cecum of group A.

lower than those in the BAY-m-1099 and control groups. Significantly decreased levels of triglycerides were also found in the Acarbose groups.

\section{DISCUSSION}

It is known that, after intake of Acarbose, human fecal microflora show increased numbers of carbohydrate-dependent bacteria and decreased numbers of protein-dependent bacteria (14). Results of the present study indicated that the cecal microflora of rats fed Acarbose-supplemented diets by feeding day (FD) 14 was characterized by significantly decreased numbers of Enterobacteriaceae and increased numbers of lecithinase-negative Clostridium spp. and Peptostreptococcus spp. By FD 28 of the Acarbose groups, the intestinal microflora showed a decrease of Enterobacteriaceae and increases of Bifidobacterium spp., lecithinase-negative Clostridium spp., Peptostreptococcus spp., and Staphylococcus spp. These findings indicated that Acarbose on FD 28 had resulted in increased gram-positive bacteria in the cecum of rats, the same as in the Acarbose feeding in humans (14). The main carbohydrate-dependent bacteria in the rat intestine were Lactobacillus spp. and Bifido- 


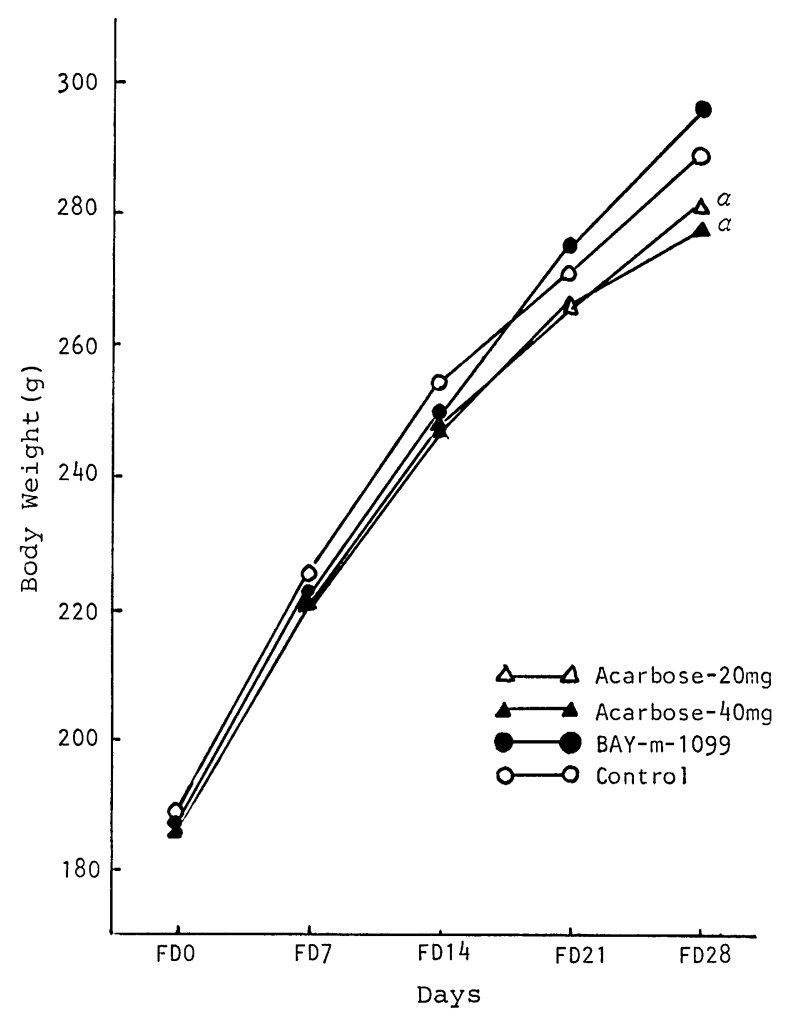

Fig. 1. Effects of Acarbose and BAY-m-1099 on body-weight gains of rats. $a$ : Statistically significant at the $P<0.001$ when compared with control.

bacterium spp. By FD 28, the higher numbers and frequency of occurrence of Bifidobacterium spp., but not Lactobacillus spp., in the cecum of rats fed Acarbose were observed. Yazawa et al (17) demonstrated that sugar sources played an important role in the intestinal proliferation of Bifidobacterium spp. in humans. The result in the present study indicates that the increased numbers of Bifidobacterium spp. are influenced by the concentrations of sugar in the intestine. It is not known why lecithinase-negative Clostridium spp. was increased by the Acarbose feeding.

The influence of BAY-m-1099 on the intestinal microflora of rats was indicated by the significantly increased number of Staphylococcus spp. Rats fed BAY-m-1099 showed more fusiform bacteria and Enterobacteriaceae than those fed Acarbose. The fecal microflora of rats fed BAY-m-1099-supplemented diets was similar to that of the controls. These changes in fecal microflora of rats fed BAY-m-1099 may be related to the absorption of BAY-m-1099 from the small intestines, especially the ileum.

The cecal weight of all rats fed Acarbose-supplemented diets in the present study was significantly higher than that of the controls and BAY-m-1099 group. The cecal weight of rats fed Acarbose diets increased dose-dependently. The sig- 

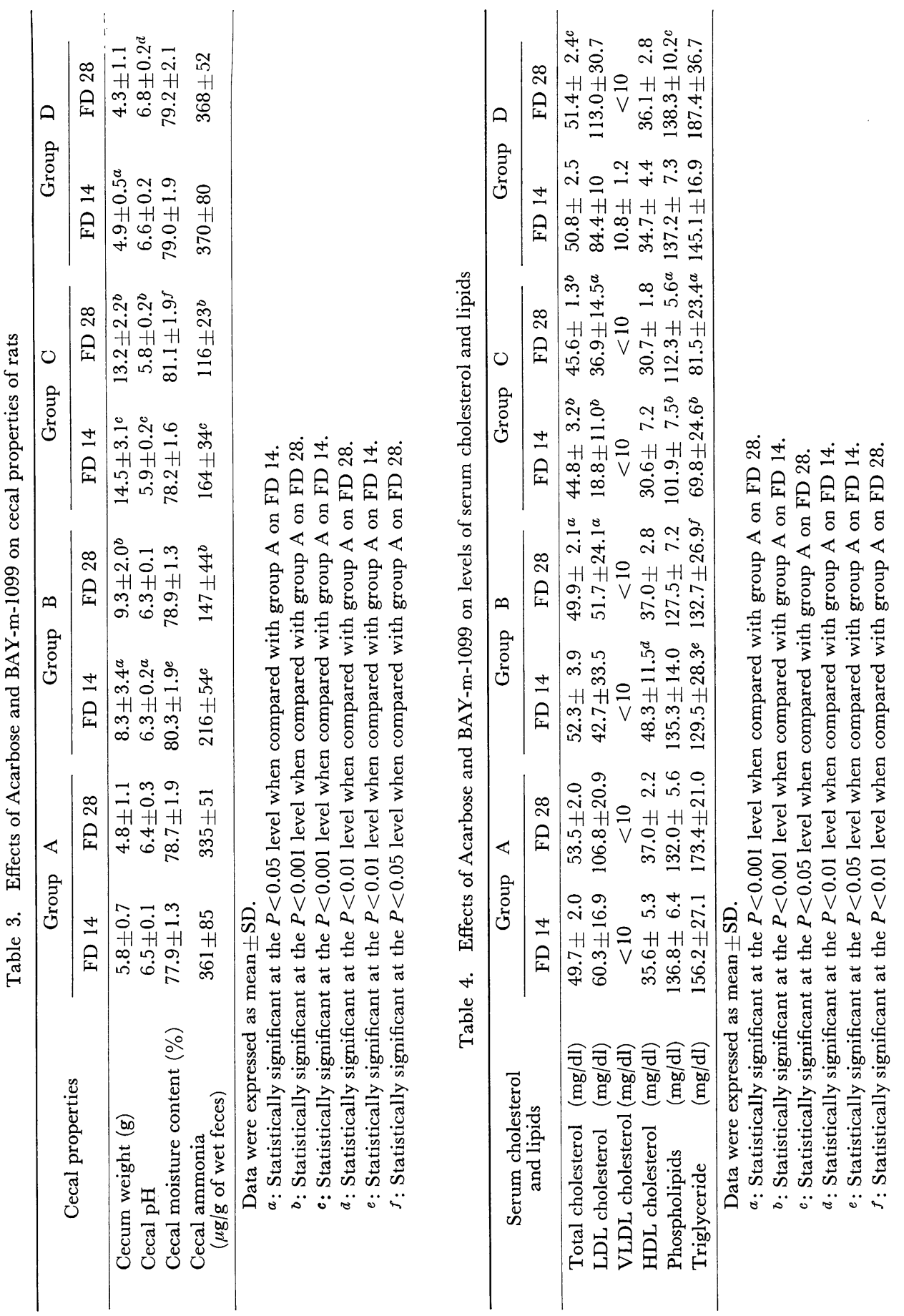
nificant reductions of cecal $\mathrm{pH}$ of rats given Acarbose diets may be related to the increased number of Bifidobacterium spp. in the cecum of these animals. The significant decrease in cecal ammonia concentration of rats fed Acarbose-supplemented diets related to the decreased number of Enterobacteriaceae and the increased number of Bifidobacterium spp. and may be due to the dilutions by the large volume of cecal contents and an increase of the water content. Different functions between Acarbose- and BAY-m-1099-supplemented diets were exerted. Briefly, no animal fed BAY-m-1099-supplemented diets had low ammonia concentration and low $\mathrm{pH}$ values in the cecum.

It is widely known that the intake volume of feed with Acarbose is dose-dependently decreased as compared with that without Acarbose $(3,11,13)$, and as a result, body-weight gains were reduced. The results of the present study were in close agreement with those of the previous study. Caspary and Kalisch reported that the highest dose of Acarbose induced diarrhea (2). However, no animal fed Acarbose (20 mg and $40 \mathrm{mg} / 100 \mathrm{~g}$ of feed) had diarrhea in this study.

On the other hand, the body weight of rats fed BAY-m-1099-supplemented diet was similar to that of the control group. Although the body-weight gains were reported to be dose-dependently reduced by BAY-m-1099-supplemented diet, bodyweight gains the same as in the control group were observed in rats fed BAY-m1099 in the present study.

It is known that the levels of serum cholesterol and triglycerides are lowered by the Acarbose supplementation. The results of the present study were in agreement with those in previous reports $(4,16)$. The levels of total cholesterol, LDL cholesterol, and triglycerides in the serum of rats fed Acarbose-supplemented diets were observed to be dose-dependently reduced. LDL cholesterol in the Acarbose group reflected the changes in total cholesterol. Our results, however, also showed that BAY-m-1099 supplementation did not decrease the levels of serum triglycerides and LDL cholesterol.

In conclusion, we have been able to show in this study that Acarbose supplementation is associated with significant changes in the composition of intestinal microflora, cecal weight, cecal $\mathrm{pH}$ values, cecal ammonia concentration, but the changes by BAY-m-1099 supplementation were not similar to those by Acarbose.

Acknowledgements. The authors thank M. Imamura, T. Oowada, A. Ozaki, and T. Yamamoto for the expert technical assistance.

\section{REFERENCES}

(1) Allain, C.C., L.S. Poon, C.S.G. Chan, W. Richmond, and P.G. Fu. 1974. Enzymatic determination of total serum cholesterol. Clin. Chem. 20: 470-475.

(2) Caspary, W.F., and H. Kalisch. 1978. Effect of $\alpha$-glucosidehydrolase inhibition on intestinal absorption of sucrose, water, and sodium in man. Gut 20:750-755.

(3) Goda, T., K. Yamada, M. Sugiyama, S. Moriuchi, and N. Hosoya. 1982. Effect of sucrose and Acarbose feeding on the development of streptozotocin-induced diabetes in the rat. J. Nutr. Sci. Vitaminol. 28: 41-56.

(4) Hillebrand, I., K. Boehme, H. Frank, and P. Berchtold. 1979. The effects of the $\alpha$-glucosidase 
inhibitor BAY g 5421 (Acarbose) on meal-stimulated elevations of circulating glucose, insulin, and triglyceride levels in man. Res. Exp. Med. (Berl.) 175: 81-86.

(5) Koditschek, L.K., and W.W. Umbreit. 1969. $\alpha$-Glycerophosphate oxidase in Streptococcus faecium F 24. J. Bacteriol. 98: 1063-1068.

(6) Mitsuoka, T., K. Ohono, Y. Benno, K. Suzuki, and K. Namba. 1976. The fecal microflora of man. V. Communication: comparison of the newly developed method with the old conventional method for the analysis of intestinal microflora. Zentralbl. Bakteriol. Parasitenkd. Infektionskr. Hyg. I Abt. Orig. A 234: 219-233.

(7) Mitsuoka, T., T. Sega, and S. Yamamoto. 1965. Eine verbesserte Methodik der qualitativen und quantitaiven Analyse der Darmflora von Menschen und Tieren. Zentralbl. Bakteriol. Parasitenkd. Infektionskr. Hyg. I Abt. Orig. A 195: 219-233.

(8) Mitsuoka, T., Y. Morishita, C. Kaneuchi, S. Yamamoto, and M. Ogata. 1969. A simple method ("Plate-in-bottle method") for the cultivation of fastidious anaerobes. Jpn. J. Microbiol. 13: 383-385.

(9) Noma, A., K. Nezu-Nakayama, M. Kita, and H. Okabe. 1978. Simultaneous determination of serum cholesterol in high- and low-density lipoproteins with use of heparin, $\mathrm{Ca}^{2+}$, and an anion-exchange resin. Clin. Chem. 24: 1504-1508.

(10) Noma, A., K. Nezu-Nakayama, Y. Ueno, and H. Shinohara. 1979. Improved method for simultaneous determination of cholesterol in high- and low-density lipoproteins. Clin. Chem. 25: $1480-1481$.

(11) Puls, W., U. Keup, H.P. Krause, L. Müller, D.D. Schmidt, G. Thomas, and E. Truscheit. 1980. Pharmacology of a glucosidase inhibitor. Front. Hormone Res. 7 : 235-247.

(12) Puls, W., U. Keup, H.P. Krause, G. Thomas, and F. Hoffmeister. 1977. Glucosidase inhibition: a new approach to the treatment of diabetes, obesity, and hyperlipoproteinaemia. Naturwissenschaften 64: 536 .

(13) Schmidt, D.D., W. Frommer, B. Junge, L. Müller, W. Wingender, and E. Truscheit. 1977. $\alpha$-Glucosidase inhibitors: new complex oligosaccharides of microbial origin. Naturwissenschaften 64: S535-S536.

(14) Shimoyama, T., S. Hori, K. Tamura, N. Tanida, M. Hosomi, and M. Wada. 1981. Effects of Acarbose on fecal microflora of hyperlipidaemic patients. Proc. 1st Int. Sym. Acarbose, p. 123136. Effects on Carbohydrate and Fat Metabolism, In W. Creutzfeldt (ed.), Excerpta Medica, Montreux.

(15) Takayama, M., S. Itoh, T. Nagasaki, and I. Tanimizu. 1977. A new enzymatic method for determination of serum choline-containing phospholipids. Clin. Chim. Acta 79: 93-98.

(16) Yamashita, K., S. Sugawara, and I. Sakairi. 1984. Effects of an $\alpha$-glucosidase inhibitor: acarbose, on blood glucose and serum lipids in streptozotocin-induced diabetic rats. Horm. Metab. Res. 16: 179-182.

(17) Yazawa, K., A. Nakajima, and Z. Tamura. 1984. Growth of bifidobacteria in adults' intestines on oral administration of sugar source, pantethine and riboflavin. Bifidobacteria Microflora 1: 39-44. 